\title{
Canadlà
}

\section{Learning guide}

\section{Mutual learning for policy impact: Insights from CORE Shaping policy and practice with intersectional gender responsive evidence (in the context of Covid-19)}

\section{Background}

On the 19 and 20 October 2021, the Institute of Development Studies hosted an online dialogue which aimed to enhance efforts to inform and influence policy, management, and practice with intersectional gender-responsive evidence by sharing learning between CORE cohort members from their approaches and experiences at country and regional levels.

The event was attended by over 30 participants from 19 partners across the CORE cohort and highlighted the experiences of CORE partners Glasswing and the Arab Reform Initiative (ARI).

This learning guide captures the practical insights and advice from the event, to help inform the practice of participants and other projects across the portfolio.

\section{Challenge: Demonstrating policy impact is often challenging}

\section{Key message: Consider different forms of impact}

Whilst instrumental impact - influencing the development of policy, practice, or services - is often the main outcome research projects seek, we were reminded that there are other ways in which our research can have an impact. These are: conceptual (contributing to the understanding of policy issues and reframing debates), building and strengthening networks, and capacity building (through technical and personal skill development).

\section{Challenge: Moving beyond gender sensitive to a gender transformative approach}

Key message: Work to change perceptions and social norms

Glasswing highlighted in their presentation the challenge of changing public perceptions where there is stigma attached to certain groups based on socio-economic characteristics in addition to gender (for example, returning migrants or informal workers). This is a challenge in many contexts, as gender is not considered an important rationale and can be a difficult barrier to overcome in achieving impact. To transform gender inequities, changing social norms on gender roles and the institutional arrangements which (re)enforce such norms through the allocation of resources would be needed. When the institutions and policy makers are not receptive, there are other ways to have an impact, such as reframing public narratives and networking, to pave alternative ways for structural policy changes.

- Engage popular media

WIEGO work with journalists with the aim of reducing the stigma attached to particular groups (such as migrants and women in the informal economy).

- Work with allies

Often, the stakeholders with the power to enact political or social change are not well aligned with your message. It can be helpful to work with allies (such as ministries of women, or gender groups) and form alliances to target the stakeholders who hold the power.

- Consider re-framing the issue

Changing the way an issue is framed can be a useful tool to help it gain support. For example, framing undernutrition as an economic issue, rather than a health issue, helped secure commitment and funding in many countries.

Consider how gender issues are currently framed. Is there an alternative that may be more favourable? 


\section{Challenge: Operating inclusively in closed policy spaces}

\section{Key message: Adapt your approach to suit the context}

- Understand political and cultural tensions

Moving to the knowledge translation phase of their programme, ARI have been convening multi-stakeholder meetings to share their research findings. However, due to political tensions, closed policy spaces, and Covid-19 restrictions in some of their focus countries, they were unable to host meetings with both government and civil society organisations (CSOs) in the same space. To address this challenge, they developed national policy dialogue guidelines and adopted a relay approach where they first brought CSOs together to create an outcome document with key messages which could then presented to policy makers at a separate meeting. This approach allowed both parties to contribute to the meetings without compromising safety.

- Engage with multiple stakeholders throughout the research

From the beginning of their research, ARI have engaged an advisory committee comprised of multiple stakeholders. This committee serves to provide feedback on the research and overall guidance, whilst also helping to facilitate access to data and leveraging networks and access to advocacy channels. Engaging with stakeholders from across sectors in this way not only allows the research itself to be adaptive and shaped by those with whom they are aiming to reach, but also aims to sensitise stakeholders and build critical networks for engaging with findings and recommendations.

\section{Challenge: Conveying complexity and nuance to multiple stakeholders} Key message: Tailor your messages for each audience

Many of the CORE projects are working across multiple countries and on a diverse range of issues, including governance, informal economy, digital transformations, migration, and social protection. Synthesising complex research programmes, particularly to policy audiences, into locally relevant practical recommendations can be a challenge.

- Understand your audience

Not every aspect of your research will resonate with every stakeholder you wish to reach. Understanding your different stakeholders is essential - how aligned they are with your work, and how influential they are in the change you wish to seek. Using a stakeholder profiling tool can help to build a picture of what each stakeholder finds important and can help you to build your strategy for engaging them.

\section{About this report}

This learning guide was produced as part of the Covid-19 Responses for Equity (CORE) Knowledge Translation Programme, which supports the translation of knowledge emerging from the CORE initiative. Supported by the International Development Research Centre, CORE brings together 21 projects to understand the socioeconomic impacts of the pandemic, improve existing responses, and generate better policy options for recovery.

The views expressed herein are those of the authors and do not necessarily represent those of IDRC or its Board of Governors, or IDS.

It is distributed under the terms of the Creative Commons Attribution 4.0 International licence (CC BY), which permits unrestricted use, distribution in any medium, provided the original authors and sources are credits and any modifications or adaptations are indicated.

(C) Institute of Development Studies 2021

DOI: 10.19088/CORE.2021.007 